FACTA UNIVERSITATIS (NIŠ)

Ser. Math. Inform. Vol. 35, No 5 (2020), 1259-1271

https://doi.org/10.22190/FUMI2005259J

\title{
STRONG CONVERGENCE THEOREM FOR UNIFORMLY L-LIPSCHITZIAN MAPPING OF GREGUS TYPE IN BANACH SPACES
}

\author{
Olilima O. Joshua, Mogbademu A. Adesanmi and Adeniran T. Adefemi
}

(C) 2020 by University of Niš, Serbia | Creative Commons Licence: CC BY-NC-ND

Abstract. In this paper, we introduced a new mapping called uniformly L-Lipschitzian mapping of Gregus type, and used the Mann iterative scheme to approximate the fixed point. A Strong convergence result for the sequence generated by the scheme is shown in real Banach space. Our result generalized and unify many recent results in this area of research. In addition, using Java (jdk 1.8.0_101), we give a numerical example to support our claim.

Key words: Mann iterative scheme; uniformly L-Lipschitzian mapping; normalized duality mapping.

\section{Introduction}

Let $E$ and $E^{*}$ be a real Banach space and its dual space respectively. The normalized duality mapping $J: E \rightarrow 2^{E^{*}}$ is defined by

$$
J(x)=\left\{f \in E^{*}:\langle x, f\rangle=\|x\|^{2}=\|f\|^{2},\|x\|=\|f\|\right\}
$$

where $\langle\cdot, \cdot\rangle$ denotes the duality pairing.

Definition 1.1. [Ofoedu E.U [13]] Let $K$ be a nonempty closed convex subset of a real Banach space $E$. The mapping $T: K \rightarrow E$ is said to be

i) nonexpansive if for all $x, y \in K$

$$
\|T x-T y\| \leqslant\|x-y\| .
$$

ii) uniformly $\mathrm{L}$-Lipschitzian if there exists $L>0$ such that, for any $x, y \in K$

$$
\left\|T^{n} x-T^{n} y\right\| \leqslant L\|x-y\|, \quad \forall n \geqslant 1 .
$$

Received December 23, 2019; accepted March 05, 2020

2020 Mathematics Subject Classification. Primary 47H10; Secondary 47H09, 54H25 
iii) asymptotically nonexpansive if there exists $k_{n} \subset[1, \infty)$ with $k_{n} \rightarrow 1$ as $n \rightarrow \infty$ such that for any given $x, y \in K$,

$$
\left\|T^{n} x-T^{n} y\right\| \leqslant k_{n}\|x-y\|, \quad \forall n>1 .
$$

iv) asymptotically pseudocontractive if there exists a sequence $k_{n} \subset[0, \infty)$ with $k_{n} \rightarrow 1$ as $n \rightarrow \infty$. and there exists $j(x-y) \in J(x-y)$ such that

$$
\left\langle T^{n} x-T^{n} y, j(x-y)\right\rangle \leqslant k_{n}\|x-y\|^{2}, \quad n \geqslant 1 .
$$

We can easily see from equations (1.2), (1.3), (1.4) that the class of asymptotically non-expansive mappings is a generalization of the class of uniformly $\mathrm{L}-$ Lipschitzian mapping. And that every asymptotically nonexpansive mappings are asymptotically pseudocontractive, the reason is shown below,

$$
\left\langle T^{n} x-T^{n} y, j(x-y)\right\rangle \leqslant\left\|T^{n} x-T^{n} y\right\|\|x-y\| \leqslant k_{n}\|x-y\|^{2}, \quad \forall n \geqslant 1 .
$$

But the converse is not always true. The example to show that the converse is not true was constructed by Rhoades [15]. The asymptotically nonexpansive mappings and the asymptotically pseudocontractive mappings were introduced by Goebel and Kirk [4] and Schu [16] respectively.

In 1980, Gregus [5] introduced what is now known as the Gregus fixed point theorem. He proved the following theorem.

Theorem 1.1. Gregus [5] Let $K$ be a closed convex subset of a Banach space $E$ and $T: K \rightarrow K$ a mapping that satisfies $\|T x-T y\| \leqslant a\|x-y\|+b\|x-T x\|+c\|y-T y\|$ for all $x, y \in K$ where $0<a<1, b, c \geqslant 0$ and $a+b+c=1$. Then $T$ has a unique fixed point.

The class of mapping introduced by Gregus [5] is a generalization of nonexpansive mapping which is a very important mapping in fixed point theorem and applications, because if $a=1, b=c=0$ then we have the mapping in (1.1), and if $a=0, b=c=\frac{1}{2}$ we have the Kannan mappings introduced by Kannan in [6]. This class of mappings have been extended by many authors in various ways and under different conditions on $T$. For results on these, see $[8,11,12,14]$ and the references therein.

The trend for uniformly L-Lipschitzian mapping and asymptotically pseudocontractive mapping is given below for better understanding the concept we intend to introduce.

In $1991 \mathrm{Schu}$ [16], proved the following result using the modified Mann iterative scheme

Theorem 1.2. Schu [16] Let $H$ be a Hilbert space, $K$ be a nonempty bounded closed convex subset of $H$ and $T: K \rightarrow K$ be a completely continuous, uniformly $L$ Lipschitzian and asymptotically pseudo-contractive mapping with a sequence $\left\{k_{n}\right\}$ satisfying the following conditions: 
(i) $k_{n} \rightarrow 1$ as $n \rightarrow 1$;

(ii) $\sum_{n=1}^{\infty} q_{n}^{2}-1<\infty$, where $q_{n}=2 k_{n}-1$.

Suppose further that $\left\{\alpha_{n}\right\}$ and $\left\{\beta_{n}\right\}$ are two sequences in $[0,1]$ such that $\epsilon<\alpha_{n}<$ $b, \quad \forall n \geqslant 1$ where $\epsilon>0$ and $b \in\left(0, L^{-2}\left[\left(1+L^{2}\right)^{1 / 2}-1\right]\right)$ are some positive numbers. For any $x_{1} \in K$, let $\left\{x_{n}\right\}$ be the iterative sequence defined by

$$
x_{n+1}=\left(1-\alpha_{n}\right) x_{n}+\alpha_{n} T^{n} x_{n}, \quad \forall n \geqslant 1 .
$$

Then $\left\{x_{n}\right\}$ converges strongly to a fixed point of $T$ in $K$.

In 2000, Chang [1] extended theorem 1.2 from Hilbert space to uniformly smooth Banach space, by proving the following theorem:

Theorem 1.3. Chang [1] Let $E$ be a real uniformly smooth Banach space, $K$ be a nonempty bounded closed convex subset of $E, T: K \rightarrow K$ be an asymptotically pseudocontractive mapping with sequence $\left\{k_{n}\right\} \subset[1,+\infty), \lim _{n \rightarrow \infty} k_{n}=1$, and $F(T)=$ $\{x \in K: T x=x\} \neq \varnothing$. Let $\alpha_{n} \subset[0,1]$ satisfying the following conditions:

(i) $\alpha_{n} \rightarrow 0$ as $n \rightarrow \infty$;

(ii) $\sum_{n=1}^{\infty} \alpha_{n}=\infty$.

For any $x_{0} \in K$, let $\left\{x_{n}\right\}$ be the iterative sequence defined by

$$
x_{n+1}=\left(1-\alpha_{n}\right) x_{n}+\alpha_{n} T^{n} x_{n}, \quad \forall n \geqslant 0,
$$

If there exists a strict increasing function $\Phi:[0, \infty) \rightarrow[0, \infty)$ with $\Phi(0)=0$ such that

$$
\left\langle T^{n} x-p, j(x-p)\right\rangle \leqslant k_{n}\|x-p\|^{2}-\Phi(\|x-p\|),
$$

for all $x \in K$ and $n \geqslant 0$, where $p \in F(T)$, then $x_{n} \rightarrow p$ as $n \rightarrow \infty$.

Recently Ofoedu [13], extended theorem 1.3 from uniformly smooth Banach space to real Banach space and he also dispensed with the boundedness condition imposed by earlier researchers, by stating and proving the following theorem:

Theorem 1.4. Ofoedu [13] Let $E$ be a real Banach space, $K$ be a nonempty closed and convex subset of $E, T: K \rightarrow K$ be a uniformly L-Lipschitzian asymptotically pseudocontractive mapping with sequence $\left\{k_{n}\right\}_{n \geqslant 0} \subset[1,+\infty), \lim _{n \rightarrow \infty} k_{n}=1$, and let $p \in F(T)=\{x \in K: T x=x\} \neq \varnothing$. Let $\alpha_{n} \subset[0,1]$ satisfying the following conditions:

(i) $\sum_{n=1}^{\infty} \alpha_{n}=\infty$;

(ii) $\sum_{n=1}^{\infty} \alpha_{n}^{2}<\infty$; 
(iii) $\sum_{n=1}^{\infty} \alpha_{n}\left(k_{n}-1\right)<\infty$

For any $x_{0} \in K$, let $\left\{x_{n}\right\}$ be the iterative sequence defined by

$$
x_{n+1}=\left(1-\alpha_{n}\right) x_{n}+\alpha_{n} T^{n} x_{n}, \quad \forall n \geqslant 0 .
$$

If there exists a strict increasing function $\Phi:[0, \infty) \rightarrow[0, \infty)$ with $\Phi(0)=0$ such that

$$
\left\langle T^{n} x-p, j(x-p)\right\rangle \leqslant k_{n}\|x-p\|^{2}-\Phi(\|x-p\|)
$$

for all $x \in K$ and $n \geqslant 0$, where $p \in F(T)$, then $x_{n} \rightarrow p$ as $n \rightarrow \infty$.

Inspired by the above results, we introduce the following concept which generalizes the class of uniformly L-Lipschitzian mappings. The mapping is defined as follows:

Definition 1.2. Let $K$ be a nonempty closed subset of a real Banach space $E$. The mapping $T: K \rightarrow E$ is said to be uniformly L-Lipschitzian mapping of Gregus type if there exists $L>0$, and the sequences $a_{n}, b_{n} \in[0, \infty)$, with $a_{n}, b_{n} \rightarrow 0$ as $n \rightarrow \infty$, such that for any $x, y \in K$,

$$
\left\|T^{n} x-T^{n} y\right\| \leqslant L\|x-y\|+a_{n}\left\|x-T^{n} x\right\|+b_{n}\left\|y-T^{n} y\right\|, \quad \forall n \geqslant 1 .
$$

If we set $a_{n}=b_{n}=0 \quad \forall n \in \mathbb{N}$, equation (1.7) is reduced to (1.2). Clearly, every uniformly L-Lipschitzian mapping is uniformly L-Lipschitaian mapping of Gregus type, but the converse is not generally true. It suffices to construct an example of a map that is uniformly L-Lipschitzian of Gregus type but not uniformly L-Lipschitzian.

Example 1.1. Let $E=\mathbb{R}$ be the set of real numbers with the usual norm, and let $K=[0, \infty)$. Consider the mapping $T: K \rightarrow K$ defined by

$$
T x=\frac{x^{3}}{4(1+x)}, \quad \forall x \in K
$$

It is easy to see that $T$ is a monotone increasing function satisfying (1.7), but $T$ does not satisfy inequality (1.2). In fact,

$$
\begin{aligned}
\left|T^{n} x-T^{n} y\right| & \leqslant|T x-T y|=\left|\frac{x^{3}}{4+4 x}-\frac{y^{3}}{4+4 y}\right|=\frac{1}{4}\left|\frac{x^{3}}{1+x}-\frac{y^{3}}{1+y}\right| \\
& =\frac{1}{4}\left|\frac{x-y+x^{3}(1+y)-x+y-y^{3}(1+x)}{(1+x)(1+y)}\right| \\
& \leqslant \frac{1}{4}\left|\frac{x-y}{(1+x)(1+y)}\right|+\left|\frac{x^{3}(1+y)-x}{4(1+x)(1+y)}\right|+\left|\frac{y-y^{3}(1+x)}{4(1+x)(1+y)}\right| \\
& \leqslant \frac{1}{4}|x-y|+\left|x-\frac{x^{3}}{4(1+x)}\right|+\left|y-\frac{y^{3}}{4(1+y)}\right| \\
& =\frac{1}{4}|x-y|+|x-T x|+|y-T y| .
\end{aligned}
$$


Therefore,

$$
\left|T^{n} x-T^{n} y\right| \leqslant \frac{1}{4}|x-y|+|x-T x|+|y-T y| .
$$

Hence, $T$ is uniformly L-Lipschitzian mapping of Gregus type where the sequences $a_{n}=$ $b_{n}=1, \forall n \in \mathbb{N}$ and $L=\frac{1}{4}$. But observe that,

$$
\frac{x^{3}}{4(1+x)}>x \quad \forall x>\frac{4+\sqrt{32}}{2},
$$

hence we have that,

$$
\left|T^{n} x-T^{n} y\right| \leqslant|T x-T y|=\left|\frac{x^{3}}{4+4 x}-\frac{y^{3}}{4+4 y}\right|>|x-y| .
$$

Thus, $T$ is not a uniformly L-Lipschitzian mapping. We can now say that the class of uniformly L- Lipschitzian mappings of Gregus type properly includes the class of uniformly L-Lipschitzian mappings. Hence, it is more important to study this class of mappings in fixed point theory and applications.

In particular, If we let $x$ to be any point in $K$ and $y \in F(T)$ then, from (1.8) we have,

$$
\left|T^{n} x-T^{n} y\right| \leqslant \frac{1}{2}|x-0|+\frac{1}{4}|x-T x|+\frac{1}{4}|0-T 0| .
$$

but

$$
\left|T^{n} x-T^{n} y\right|=\left|T^{n} x-0\right| \leqslant|T x-0|=\left|\frac{x^{3}}{4+4 x}-0\right|>|x-0|,
$$

for $x>\frac{4+\sqrt{32}}{2}, y=0 \in F(T)$.

It is our aim in this paper to consider the iterative scheme in (1.6) and prove a strong convergence theorem for the newly introduced uniformly L-Lipschitzian mappings of Gregus type to a unique fixed point in real Banach spaces.

\section{Preliminaries}

We shall need the following Proposition and lemmas in the main theorem.

Proposition 2.1. Let $K$ be a nonempty closed convex subset of a Banach space and $T: K \rightarrow K$ be a Uniformly L-Lipschitzian mapping of Gregus Type with $a_{n} \in\left(0, \frac{1}{2}\right)$ such that $a_{n} \rightarrow 0$ as $n \rightarrow \infty$. Then for any $x_{0} \in K$, let $\left\{x_{n}\right\}$ be an iterative sequence defined by

$$
x_{n+1}=\left(1-\alpha_{n}\right) x_{n}+\alpha_{n} T^{n} x_{n}
$$

for all $n \geqslant 0$, there exists an $M>0$ such that for $p \in F(T)$, the following hold:

i.) $\left\|T^{n} x_{n}-x_{n}\right\| \leqslant M\left\|x_{n}-p\right\|$

ii.) $\left\|T^{n} x_{n+1}-x_{n+1}\right\| \leqslant M\left\|x_{n+1}-p\right\|$ 
Proof. Since $T$ is uniformly L-Lipschitzian mapping of Gregus Type, we have,

$$
\begin{aligned}
\left\|x_{n}-T^{n} x_{n}\right\| & \leqslant\left\|x_{n}-p\right\|+\left\|T^{n} x_{n}-p\right\|=\left\|x_{n}-p\right\|+\left\|T^{n} x_{n}-T^{n} p\right\| \\
& \leqslant\left\|x_{n}-p\right\|+L\left\|x_{n}-p\right\|+a_{n}\left\|x_{n}-T^{n} x_{n}\right\|+b_{n}\left\|p-T^{n} p\right\| \\
& =(1+L)\left\|x_{n}-p\right\|+a_{n}\left\|x_{n}-T^{n} x_{n}\right\| \\
& \leqslant \frac{(1+L)}{1-a_{n}}\left\|x_{n}-p\right\| .
\end{aligned}
$$

Since, $a_{n} \in[0,1 / 2)$ we have that, $-a_{n}>-\frac{1}{2}$. Hence, $1-a_{n}>1-\frac{1}{2}$, this implies that

$$
\frac{1}{1-a_{n}}<2
$$

Therefore,

$$
\frac{(1+L)}{1-a_{n}}<2(1+L)
$$

Let $M=2(1+L)>1,(2.1)$ becomes

$$
\left\|T^{n} x_{n}-x_{n}\right\| \leqslant M\left\|x_{n}-p\right\| .
$$

Using similar procedure we can easily get that

$$
\left\|T^{n} x_{n+1}-x_{n+1}\right\| \leqslant M\left\|x_{n+1}-p\right\| .
$$

This completes the proof.

Lemma 2.1. Mogbademu [9] Let $E$ be a normed linear space then for all $x, y \in E$ and for all $j(x+y) \in J(x+y)$, the following inequality holds:

$$
\|x+y\|^{2} \leqslant\|x\|^{2}+2\langle y, j(x+y)\rangle .
$$

Lemma 2.2. C. Moore and B.V Nnoli [10] Let $\Phi:[0,+\infty) \rightarrow[0,+\infty)$ be a strictly increasing function with $\Phi(0)=0$ and let $\left\{\theta_{n}\right\},\left\{\lambda_{n}\right\},\left\{\sigma_{n}\right\}$ be any nonnegative real sequences such that $\sigma_{n}=o\left(\lambda_{n}\right), \sum_{i=0}^{\infty} \lambda_{n}=\infty$, and $\lim _{n \rightarrow \infty} \lambda_{n}=0$. Suppose that

$$
\theta_{n+1}^{2} \leqslant \theta_{n}^{2}-\lambda_{n} \Phi\left(\theta_{n+1}\right)+\sigma_{n}, \quad n \geqslant 1,
$$

then $\lim _{n \rightarrow \infty} \theta_{n}=0$.

\section{The Main Result}

Theorem 3.1. Let $E$ be a real Banch space, $K$ be a nonempty closed convex subset of $E, T: K \rightarrow K$ be a Uniformly L-Lipschitzian mapping of Gregus Type with $F(T) \neq \varnothing$ where $F(T)=\{x \in K: T x=x\}$ and $p \in F(T)$. Let $\left\{k_{n}\right\} \in[0, \infty)$ be a sequence of real numbers such that $k_{n} \rightarrow 1$ as $n \rightarrow \infty$, and let $\alpha_{n} \in[0,1]$ satisfying the following: 
i) $\alpha_{n} \rightarrow 0$ as $n \rightarrow \infty$

ii) $\sum_{n=0}^{\infty} \alpha_{n}=\infty$;

For any $x_{0} \in K$, define a sequence $\left\{x_{n}\right\}$ by

$$
x_{n+1}=\left(1-\alpha_{n}\right) x_{n}+\alpha_{n} T^{n} x_{n}
$$

for all $n \geqslant 0$. If there exists a strictly increasing function $\Phi:[0,+\infty) \rightarrow[0,+\infty)$ with $\Phi(0)=0$ such that

$$
\left\langle T^{n} x-p, j(x-p)\right\rangle \leqslant k_{n}\|x-p\|^{2}-\Phi(\|x-p\|)
$$

for all $x \in K$. Then

i) $\left\{x_{n}\right\}_{n \geqslant 0}$ is bounded;

ii) $x_{n} \rightarrow p$ as $n \rightarrow \infty$ where $p$ is a unique fixed point of $T$.

Proof. This proof shall be divided into two steps. In step 1, we will show boundedness, while in step 2 we will show that the iterative sequence $\left\{x_{n}\right\}_{n=0}^{\infty}$ converges strongly to the unique fixed point of $T$ say $p$.

Step 1: Let $k=\sup \left\{k_{n}: n \geqslant 1\right\}$, since $T$ is Uniformly L-Lipschitzian of Gregus Type and $\Phi:[0, \infty) \rightarrow[0, \infty)$ is a strictly increasing real valued function with $\Phi(0)=0$, for $x \in K, p \in F(T)$ we obtain

$$
\Phi(\|x-p\|) \leqslant\left(k+L+\alpha_{n} M\right)\|x-p\|^{2} .
$$

Taking limit as $n \rightarrow \infty$ we have

$$
\Phi(\|x-p\|) \leqslant(k+L)\|x-p\|^{2} .
$$

Assume that $x_{1} \neq T x_{1}$ for some $x_{1} \in K$ such that

$$
(k+L)\left\|x_{1}-p\right\|^{2} \in R(\Phi),
$$

we denote that $a_{0}=(k+L)\left\|x_{1}-p\right\|^{2}$, where $R(\Phi)$ is the range of $\Phi$. Indeed, if $\Phi(a) \rightarrow \infty$ as $a \rightarrow \infty$, then $a_{0} \in R(\Phi)$; if $\sup \{\Phi(a): a \in[0, \infty)\}=a_{1}<+\infty$ with $a_{1}<a_{0}$, then for $p \in K$, there exists a sequence $\left\{u_{n}\right\}$ in $K$ such that $u_{n} \rightarrow p$ as $n \rightarrow \infty$ with $u_{n} \neq p$, thus there exists an $n_{0} \in \mathbb{N}$ such that

$$
(k+L)\left\|u_{n}-p\right\|^{2}<\frac{a_{1}}{2}
$$

for $n \geqslant n_{0}$. We redefine $x_{1}=u_{n_{0}}$ and $(k+L)\left\|x_{1}-p\right\|^{2} \in R(\Phi)$.

Set $R=\Phi^{-1}\left(a_{0}\right)$. Then we obtain $\left\|x_{1}-p\right\| \leqslant R$.

Denote

$$
B_{1}=\{x \in K:\|x-p\| \leqslant R\}, B_{2}=\{x \in K:\|x-p\| \leqslant 2 R\}
$$


Now, we show that $x_{n} \in B_{1}$ using mathematical induction for any $n \geqslant 1$. If $n=1$, then $x_{1} \in B_{1}$. Suppose that thr result is true for some $n$, that is $x_{n} \in B_{1}$. Now we show that $x_{n+1} \in B_{1}$. Suppose that, $x_{n+1} \notin B_{1}$, that is, $x_{n+1}>R$. Denote

$$
\tau_{0}=\min \left\{1, \frac{1}{2 M}, \frac{1}{2 L M}, \frac{\Phi(R)}{16 R(M(l+3) R}, \frac{\Phi(R)}{16 R^{2}}\right\} .
$$

Since $a_{n}, b_{n}, \alpha_{n}$ and $k_{n}-1 \rightarrow 0$ as $n \rightarrow \infty$. We can let $0 \leqslant a_{n}, b_{n}, \alpha_{n}, k_{n}-1 \leqslant \tau_{0}$ for any $n \geqslant 1$. We obtain the following:

$$
\begin{aligned}
\left\|x_{n+1}-p\right\| & \leqslant\left\|x_{n}-p\right\|+\alpha_{n}\left(T^{n} x_{n}-x_{n}\right) \\
& \leqslant R+\alpha_{n} M R \\
& \leqslant 2 R
\end{aligned}
$$

Using Proposition 2.1 we have the following,

$$
\begin{aligned}
\left\|T^{n} x_{n}-T^{n} x_{n+1}\right\| & \leqslant L \alpha_{n}\left\|x_{n}-x_{n+1}\right\|+a_{n}\left\|x_{n}-T^{n} x_{n}\right\|+b_{n}\left\|x_{n+1}-T^{n} x_{n+1}\right\| \\
& \leqslant\left(L \alpha_{n}+a_{n}\right) M\left\|x_{n}-p\right\|+b_{n} M\left\|x_{n+1}-p\right\| \\
& \leqslant\left(L \alpha_{n}+a_{n}\right) M R+2 b_{n} M R \\
& \leqslant \tau_{0} M R(L+3) \\
& \leqslant \frac{\Phi(R)}{16 R} .
\end{aligned}
$$

Let us consider the following estimate, using Lemma 2.1

$$
\begin{aligned}
\left\|x_{n+1}-p\right\|^{2}= & \left\|\left(1-\alpha_{n}\right) x_{n}+\alpha_{n} T^{n} x_{n}-p\right\|^{2} \\
= & \left\|\left(1-\alpha_{n}\right)\left(x_{n}-p\right)+\alpha_{n}\left(T^{n} x_{n}-p\right)\right\|^{2} . \\
\leqslant & \left(1-\alpha_{n}\right)^{2}\left\|x_{n}-p\right\|^{2}+2 \alpha_{n}\left\langle T^{n} x_{n}-p, j\left(x_{n+1}-p\right)\right\rangle \\
= & \left(1-\alpha_{n}\right)^{2}\left\|x_{n}-p\right\|^{2}+2 \alpha_{n}\left\langle T^{n} x_{n}-T^{n} x_{n+1}, j\left(x_{n+1}-p\right)\right\rangle \\
& +2 \alpha_{n}\left\langle T^{n} x_{n+1}-p, j\left(x_{n+1}-p\right)\right\rangle \\
\leqslant & \left(1-\alpha_{n}\right)^{2}\left\|x_{n}-p\right\|^{2}+2 \alpha_{n}\left\|T^{n} x_{n}-T^{n} x_{n+1}\right\|\left\|x_{n+1}-p\right\| \\
& +2 \alpha_{n}\left[k_{n}\left\|x_{n+1}-p\right\|^{2}-\Phi\left(\left\|x_{n+1}-p\right\|\right)\right] \\
\leqslant & \left(1-\alpha_{n}\right)^{2} R^{2}+2 \alpha_{n} k_{n}\left\|x_{n+1}-p\right\|^{2}+ \\
& 2 \alpha_{n} \frac{\Phi(R)}{16 R} 2 R-2 \alpha_{n} \Phi(R) .
\end{aligned}
$$

Since $\alpha_{n}, k_{n} \rightarrow 1$ as $n \rightarrow \infty$. Thus, without loss of generality, we let $1-2 \alpha_{n} k_{n}>0$ for any $n \geqslant 1$, since

$$
\frac{1}{1-2 \alpha_{n} k_{n}} \leqslant 1+\frac{2 \alpha_{n} k_{n}}{1-2 \alpha_{n} k_{n}}
$$


from (3.6) we have that

$$
\begin{aligned}
\left\|x_{n+1}-p\right\|^{2} \leqslant & R^{2}+\frac{2 \alpha_{n}}{1-2 \alpha_{n} k_{n}}\left[\left(k_{n}-1\right)+\frac{\alpha_{n}}{2}\right] R^{2}+ \\
& \frac{\alpha_{n} \Phi(R)}{4\left(1-2 \alpha_{n} k_{n}\right)}-\frac{2 \alpha_{n}}{1-2 \alpha_{n} k_{n}} \Phi(R) \\
\leqslant & R^{2}+\frac{\alpha_{n} \Phi(R)}{4\left(1-2 \alpha_{n} k_{n}\right)}+\frac{\alpha_{n} \Phi(R)}{4\left(1-2 \alpha_{n} k_{n}\right)}-\frac{2 \alpha_{n}}{1-2 \alpha_{n} k_{n}} \Phi(R) \\
= & R^{2}-\frac{3 \alpha_{n}}{2\left(1-2 \alpha_{n} k_{n}\right)} \Phi(R) \\
\leqslant & R^{2},
\end{aligned}
$$

which is a contradiction. Hence, the sequence $\left\{x_{n}\right\}$ is bounded.

Step 2: Here, we intend to show that $x_{n}$ converges uniquely to $p \in F(T)$. Firstly, let us show that $p$ is unique.

Now, we show that $p$ is unique. Suppose for contradiction there exists $p, q \in$ $F(T)$, where $p \neq q$, such that the sequence $\left\{x_{n}\right\}$ converges to $p, q$ hence, we have that,

$$
\begin{aligned}
\|p-q\| & =\left\|T^{n} p-T^{n} q\right\| \\
& \leqslant L\|p-q\|+a_{n}\left\|p-T^{n} p\right\|+b_{n}\left\|q-T^{n} q\right\| \\
& =L\|p-q\|
\end{aligned}
$$

Therefore,

$$
0 \leqslant\|p-q\| \leqslant 0
$$

Hence, $p=q$ is a contradiction.

Next, we show that the sequence $\left\{x_{n}\right\}$ converge to a unique fixed point $p$ of $T$.

Since $\left\|x_{n}-p\right\|$ is bounded, there exists $M_{*}>0$ such that $\left\|x_{n}-p\right\|^{2}<M_{*}$. Hence, from equation (3.5) we have

$$
\begin{aligned}
\left\|x_{n+1}-p\right\|^{2} \leqslant & \left(1-\alpha_{n}\right)^{2}\left\|x_{n}-p\right\|^{2}+2 \alpha_{n}\left\|T^{n} x_{n}-T^{n} x_{n+1}\right\|\left\|x_{n+1}-p\right\| \\
& +2 \alpha_{n}\left[k_{n}\left\|x_{n+1}-p\right\|^{2}-\Phi\left(\left\|x_{n+1}-p\right\|\right)\right] \\
\leqslant & \left\|x_{n}-p\right\|^{2}-\alpha_{n} \Phi\left(\| x_{n+1}-p\right) \| \\
& +2 \alpha_{n}\left(k_{n}-1\right) M_{*}^{2}+\alpha_{n}^{2} M_{*}^{2}+2 \alpha_{n} M_{*} M\left(L \alpha_{n}+a_{n}+b_{n}\right) M_{*}
\end{aligned}
$$

Comparing Lemma 2.2 with (3.9) we can let $\theta_{n+1}^{2}=\left\|x_{n+1}-p\right\|^{2}, \theta_{n}^{2}=\left\|x_{n}-p\right\|^{2}, \lambda_{n}=$ $\alpha_{n}$ and $\sigma_{n}=2 \alpha_{n}\left(k_{n}-1\right) M_{*}^{2}+\alpha_{n}^{2} M_{*}^{2}+2 \alpha_{n} M_{*} M\left(L \alpha_{n}+a_{n}+b_{n}\right) M_{*}$. From condition (i), we have that $\sum_{n=1}^{\infty} \lambda_{n}=\sum_{n=1}^{\infty} \alpha_{n}=\infty$, therefore, $\left\|x_{n}-p\right\| \rightarrow 0$ as $n \rightarrow \infty$.

Example 3.1. Consider $T=\frac{x^{3}}{4+4 x}$, we have shown in Example 1.1, that $T$ is uniformly $\mathrm{L}-$ Lipschitzian of Gregus type, and we can easily check that the fixed point of $T$ is $p=0$. 
Now, take $\alpha_{n}=1 / 2$, and the initial guess value $x_{0}=0.5,1.0,1.5$ and 2.0. In table 3.1 and figure 3.1, we give a numerical example using Java 2.7, to support our claim that the sequence $x_{n}$ converges uniquely to its fixed point $p=0$ for the uniformly L-Lipschitzian mapping of Gregus type $T$.

Table 3.1: Numerical Example for the uniformly L-Lipschitzian mapping of Gregus type using the iteration in $(1.6), T x=\frac{x^{3}}{4(1+x)}$.

\begin{tabular}{ccccc}
\hline$S / N$ & & Modified & Mann Iteration & \\
\hline 0 & 0.5 & 1 & 1.5 & 2 \\
1 & 0.260416667 & 0.5625 & 0.91875 & 1.333333333 \\
2 & 0.131959801 & 0.295488281 & 0.509897366 & 0.793650794 \\
3 & 0.066233649 & 0.150233556 & 0.265923844 & 0.43166398 \\
4 & 0.033150889 & 0.075485267 & 0.134818761 & 0.222854726 \\
5 & 0.016579852 & 0.037792625 & 0.0676793 & 0.112558723 \\
$\vdots$ & $\vdots$ & $\vdots$ & $\vdots$ & $\vdots$ \\
25 & $1.58 \mathrm{E}-08$ & $3.61 \mathrm{E}-08$ & $6.46 \mathrm{E}-08$ & $1.08 \mathrm{E}-07$ \\
26 & $7.91 \mathrm{E}-09$ & $1.80 \mathrm{E}-08$ & $3.23 \mathrm{E}-08$ & $5.39 \mathrm{E}-08$ \\
27 & $3.95 \mathrm{E}-09$ & $9.01 \mathrm{E}-09$ & $1.62 \mathrm{E}-08$ & $2.69 \mathrm{E}-08$ \\
28 & $1.98 \mathrm{E}-09$ & $4.51 \mathrm{E}-09$ & $8.08 \mathrm{E}-09$ & $1.35 \mathrm{E}-08$ \\
$\vdots$ & $\vdots$ & $\vdots$ & $\vdots$ & $\vdots$ \\
55 & $1.47 \mathrm{E}-17$ & $3.36 \mathrm{E}-17$ & $6.02 \mathrm{E}-17$ & $1.00 \mathrm{E}-16$ \\
56 & $7.36 \mathrm{E}-18$ & $1.68 \mathrm{E}-17$ & $3.01 \mathrm{E}-17$ & $5.02 \mathrm{E}-17$ \\
57 & N/A & $8.40 \mathrm{E}-18$ & $1.50 \mathrm{E}-17$ & $2.51 \mathrm{E}-17$ \\
58 & N/A & N/A & $7.52 \mathrm{E}-18$ & $1.25 \mathrm{E}-17$ \\
59 & N/A & N/A & N/A & $6.27 \mathrm{E}-18$ \\
\hline
\end{tabular}




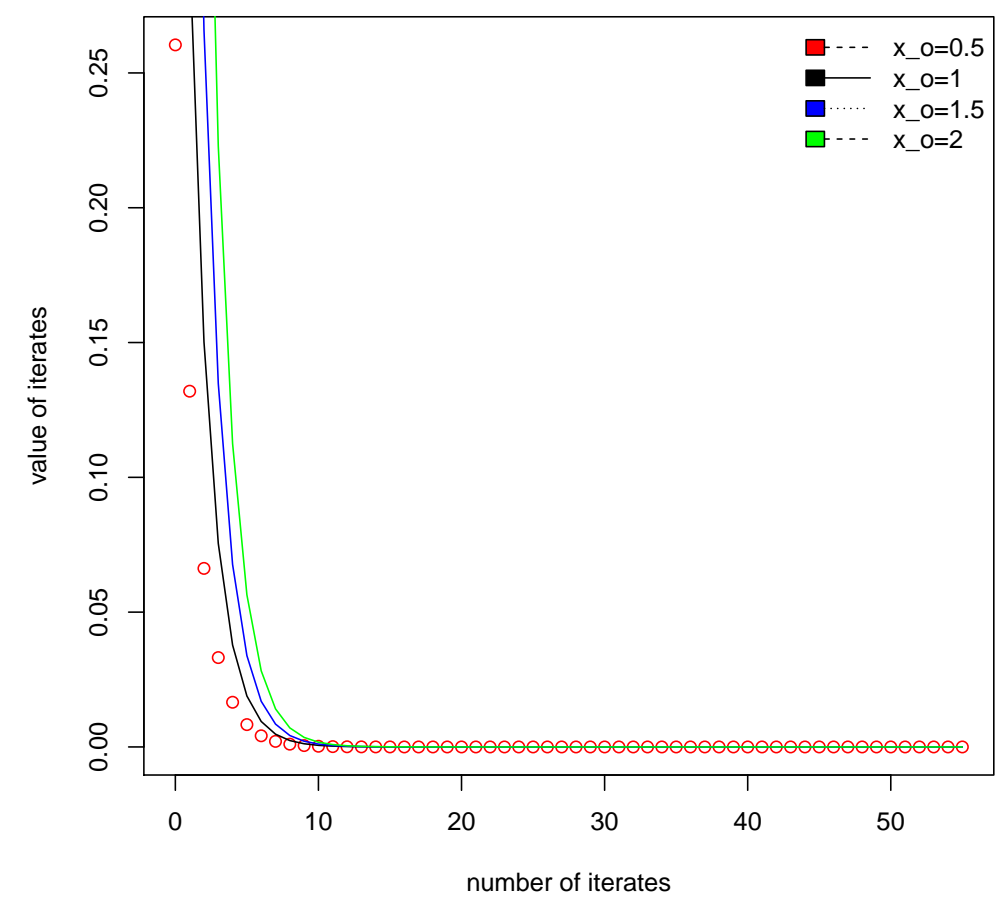

FIG. 3.1: Convergence behaviour of Modified Mann iteration process to the fixed point $p=0$ with initial guess values taken at $x_{0}=0.5,1.0,1.5$ and 2.0.

\section{Competing Interest}

The authors declares that they have no competing interest.

\section{Acknowledgments}

The first and third authors will like to appreciate the second author for his mentorship. In addition, the authors appreciates the reviewer for his observations.

\section{REF E R E N C E S}

1. S. S. Chang. Some results for asymptotically pseudocontractipings Proc. Amer. Math. Soc. 129 (2000), 845-853.

2. S. S. Chang, Y. J. Cho and J. K Kim. Some results for Uniformly Lipschitzian mappings in Banach spaces. App. Math. Letters. 22 (2009), 121-125. 
3. S. Deimling. Non linear Functional Analysis. Springer, Berlin, (1980)

4. K. Goebel and W. A. KIRK. A fixed point theorem for asymptotically nonexpansive mappings. Proc. Amer. Math. Soc. 35 (1972), 171-174.

5. M. Gregus. A fixed point theorem in Banach space. Bollettindella Unione Matematica Italiana A Vol. 5 (1980), pp. 193-198.

6. R, Kannan. Some results on fixed points. Bull. Calcutta Math. Soc. 60 (1968), 71-76.

7. W. R. Mann. Mean value methods in iteration. Proc. Amer. Math. Soc. 4 (1953), 506-510.

8. A. K. Marwan, A. Amini-Harandi and N Hussain. A generalization of a Gregus fixed point in metric spaces. Journal of Applied Math. Vol. 2014, Article ID 580297, 5 pages, 2014, https://doi.org/10.1155/2014/580297

9. A. A. Mogbademu. Fixed points of nearly weak uniformly L-Lipschitzian mappings in real Banach spaces. CREAT. MATH. INFORM. 27 (2018), No. 1, $63-70$

10. C. Moore and B. V. NNOLI. Iterative solution of nonlinear equations involving setvalued uniformly accretive operators. Comput. Math. Appl. 42 (2001), 131-140.

11. S. MorAdi and A. FARAJZADEH. On Olaleru's open problem on Gregus fixed point theorem. Journal of Global Optimization. Vol. 56, no. 4 (2013), pp. 1689-1697.

12. R,N Mukherjee and V. Verma. A note on a fixed point theorem of Gregus, Mathematica Japonica. Vol. 33 (1988), No. 5, pp. 745-749.

13. E. U. Ofoedu. Strong Convergence theorem for Uniformly L-Lipschitzian asymtotically nonexpansive mapping in Banach space. J. Math. Anal. Appl. 321 (2006), 722728.

14. J. O. Olaleru and H. Akewe. An extenson of Gregus fixed point theorem. Fixed Point Theory and Applications. Volume 2007, Article ID 78628, 8 pages

15. B. E. Rhondes. A comparison of various definition of contractive mappings, Trans. Amer. Math. Soc. 226 (1977), 257-290.

16. J. ScHU. Iterative construction of fixed point of asymptotically nonexpansive mappings. J. Math. Anal. Appl. 158 (1991), 407-413.

17. Z. XU and G. Lv: Strong convergence theorems for uniformly L-Lipschitzian asymptotically pseudocontractive mappings in Banach spaces. J. of Inequalities and Applications. 2013, 2013:79.

Olilima O. Joshua

Faculty of Science

Department of Mathematical Sciences

Augustine University Ilara-Epe

Lagos, Nigeria

P. M. Box 1010

joshua.olilima@augustineuniversity.edu.ng

Mogbademu A. Adesanmi

Faculty of Science

Department of Mathematical Sciences 
Augustine University Ilara-Epe

Lagos, Nigeria

P. M. Box 1010

amogbademu@unilag.edu.ng

Adeniran T. Adefemi

Faculty of Science

Department of Statistics

University of Ibadan

Oyo State, Nigeria

at .adeniran@mail.ui.edu.ng 